Claremont Colleges

Scholarship@ Claremont

All HMC Faculty Publications and Research

HMC Faculty Scholarship

$1-1-2004$

\title{
Optical coherence microscopy for the evaluation of a tissue-engineered artificial cornea
}

Elizabeth J.; Haskell, Richard C.; Petersen, Daniel C. Orwin

Harvey Mudd College

S Lee

C Raub

T Icenogle

MArman

See next page for additional authors

\section{Recommended Citation}

This paper first appeared as E. J. Orwin, S. Lee, C. Raub, T. Icenogle, M. Arman, A. Cho, R. Lovec, A. Malone, R. C. Haskell, B. M. Hoeling, D. C. Petersen, "Optical coherence microscopy for the evaluation of a tissue-engineered artificial cornea," Proceedings of the 26th Annual International Conference of the Ieee Engineering In Medicine and Biology Society, Vols 1-7, 26, (2004):1218-1221, and may be found on the publisher's website at http://ieexplore.iee.org/stamp/stamp.jsp?tp=\&arnumber=1403388\&isnumber=30462.

This Article is brought to you for free and open access by the HMC Faculty Scholarship at Scholarship @ Claremont. It has been accepted for inclusion in All HMC Faculty Publications and Research by an authorized administrator of Scholarship @ Claremont. For more information, please contact scholarship@cuc.claremont.edu. 


\section{Authors}

Elizabeth J.; Haskell, Richard C.; Petersen, Daniel C. Orwin; S Lee; C Raub; T Icenogle; M Arman; A Cho; R Lovec; A Malone; and BM Hoeling 


\title{
Optical Coherence Microscopy for the Evaluation of a Tissue-Engineered Artificial Cornea
}

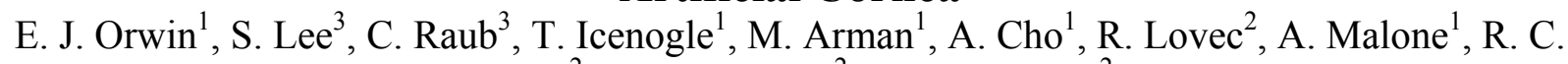 \\ Haskell $^{2}$, B. M. Hoeling ${ }^{2}$, D. C. Petersen ${ }^{2}$, \\ ${ }^{1}$ Department of Engineering, Harvey Mudd College, Claremont, CA, USA \\ ${ }^{2}$ Department of Physics, Harvey Mudd College, Claremont, CA, USA \\ ${ }^{3}$ Department of Biology, Harvey Mudd College, Claremont, CA, USA
}

\begin{abstract}
A transparent artificial cornea derived from biological material is the ultimate goal of corneal research. Attempts at artificial corneal constructs produced from synthetic polymers have proved unsuccessful due to lack of biocompatibility and ability to integrate into the tissue. We have designed a corneal model derived from collagenous biological materials that has several advantages: it has low antigenicity and therefore small chance of eliciting an immune reaction, it can be broken down by the body's own cells and gradually replaced over time by natural materials, and it may contain signaling information for native cells, thereby inducing normal phenotype and behavior. In addition, a transparent corneal model has the potential to be used for testing of novel ophthalmic drugs or gene therapy approaches, eliminating the need for animal testing. We have used an optical coherence microscope (OCM) to evaluate both the structure of our tissue constructs over time in culture and the optical properties of the tissue itself. This imaging technique promises to be an important diagnostic tool in our efforts to understand the influence of mechanical forces, cell phenotype, and soluble factors on the transparency of corneal tissue.
\end{abstract}

Keywords - Cornea, optical coherence microscopy, optical coherence tomography, tissue engineering,

\section{INTRODUCTION}

Corneal opacification, or loss of transparency, is a leading cause of blindness in the world. Causes of corneal blindness include edema, scarring, ulcers, burns, perforations or abnormal shape due to disease, metabolic disorders, infections or trauma [1,2]. Current solutions to the problem of corneal blindness include keratoplasty (corneal transplants) and synthetic keratoprostheses (implants). Concerns about disease transmission and tissue availability, as well as poor prognosis with certain diseases limits the applicability of keratoplasty. In addition, corneal transplants can still lead to many complications, including tissue rejection. Most problems with keratoprostheses occur at the implant/tissue interface and reflect the lack of biocompatibility of the material [3].

There is currently a clinical need for an improved understanding of corneal wound healing mechanisms in order to solve corneal haze problems associated with LASIK procedures [4] and outcomes in corneal transplants [5]. It would be highly desirable to develop a biodegradable scaffold that could serve as a wound healing bandage for corneal perforations and, ultimately, as an alternative to existing keratoprostheses. As an alternative to conventional polymers for the construction of such a scaffold, we have chosen a porous biodegradable polymer for the support of corneal cell infiltration and migration. Specifically, we are using a type I collagen sponge matrix produced with optimal pore size for cellular infiltration [6] from a bovine dermal source. Advantages to using such a polymer include the fact that cells have attachment proteins for collagen, therefore potentially relaying signaling information for the corneal cells; collagen is a native protein in the cornea and can be broken down and replaced by normal wound healing mechanisms; and the biodegradation rate of the collagen scaffold can be modulated in vivo.

In order to achieve a useful substrate for the study of corneal wound healing or for an artificial corneal replacement, it is necessary to understand the light transmission properties of the tissue construct. Specifically, it is important to understand the influence of corneal fibroblast cell remodeling during wound healing on the transparency of a tissue construct. Most studies of tissueengineered corneal equivalents to date have not evaluated light transmission properties of the tissue. Some studies offer qualitative observations of the transparency of their tissue constructs but have not evaluated light transmission directly. In one study, transparency of a corneal equivalent was evaluated by placing it on a white sheet of paper and evaluating the ability to observe the text through the tissue construct [7]. Standard methods for the measurement of the transparency of intact corneas have involved mounting the specimen in a holder and shining light over the range of visible wavelengths through the sample using a system designed for this purpose [8]. The main drawbacks to these methods are the complicated nature of the optical setup and the fact that samples must be sacrificed from sterile culture. In previous studies done at the University of Minnesota, transparency of the collagen sponge/cell constructs was evaluated over time in sterile culture using an inverted microscope. Light transmission through the samples was estimated using optical density images through the samples versus through cell media alone. Using this method with a band pass filter for $700 \mathrm{~nm}$ light, we found that rabbit corneas transmitted $98 \%$ of incident light, collagen sponge constructs transmitted approximately $34 \%$ of transmitted light, and collagen gels transmitted only about $5 \%$ of the light. By augmenting the collagen sponge matrix with chondroitin sulfate (a proteoglycan found in the native cornea) we were able to achieve a light transmission value of $57 \%$. [9]

These methods provided an overall indication of light transmission through our tissue, but we were not able to 
observe what factors were influencing light scattering directly. Corneal transparency is influenced by both tissue structure and cellular phenotype. The collagen structure within the intact cornea is extremely regular, creating an optically homogeneous medium. In addition, it has recently been determined that there is a cellular contribution to transparency. $30 \%$ by mass of the water soluble protein in quiescent rabbit keratocytes is comprised of the "corneal crystallins" transketolase (TKT) and aldehyde dehydrogenase 1 (ALDH1) [10]. It has been discovered that there is at least a $50 \%$ reduction in expression levels of these proteins when corneal haze following injury is present (and cells have been activated to the repair or myofibroblast phenotype). It is hypothesized from these results that these two proteins act similarly to crystallin proteins in the lens of the eye - that is, they aggregate in the cell cytoplasm and contribute to index matching of the cytosol with the surrounding extracellular environment. As further evidence for this theory, Jester and colleagues have shown that light scatter from normal keratocytes in a transparent cornea comes entirely from the nucleus of the cell - the cell body does not scatter light. [10]

In this paper we present our preliminary results of the application of OCM to study both the matrix structure and the overall transparency of our corneal tissues. The OCM provides a method for observing the amount of backscattered light in our tissue as well as structural changes over time in sterile culture.

\section{Tissue Constructs}

\section{Methodology}

Collagen sponges augmented with chondroitin sulfate were placed in specially designed bioreactor chambers, porous side up and hydrated with $150 \mu \mathrm{L}$ of cell culture media. Human corneal fibroblasts were pipetted onto the sponge surface at a density of 50,000 cells $/ \mathrm{cm}^{3}$ as per previous studies in our lab and of corneal fibroblasts cultured on collagen gels [11]. All samples were placed at $37^{\circ} \mathrm{C}$ for one hour to facilitate cell attachment to the collagen matrix and then cell media was added to submerge the cultures. Fresh media was added to the cultures every other day for the duration of the culture period. Optical properties of all samples were assessed with the OCM, and tissues were sacrificed for histological analysis at the end of the culture period.

\section{Optical Coherence Microscopy}

Our optical coherence microscope [12] allows us to obtain three-dimensional images of our tissue samples. Using a superluminescent diode with a wavelength of 850 $\mathrm{nm}$, the OCM measures the amount of light backscattered from each volume element (voxel). Through a coherence gate, only light coming from a certain depth in the sample is admitted. Typically an x-y plane of $100 \times 100$ voxels is scanned, and then the sample head is stepped down in depth and the next $\mathrm{x}-\mathrm{y}$ plane is scanned (en face scanning). For every plane scan, the position of the reference mirror is adjusted to preserve the lateral resolution of $5 \mu \mathrm{m}$ (focus tracking). The depth resolution, determined by the coherence length of the light source, is $15 \mu \mathrm{m}$ in air or 11 $\mu \mathrm{m}$ in tissue. For the display of our 3D data sets, we use the software AVS Express that allows us to volume render, rotate, and crop our images and to perform statistical analyses of the voxel contents. Samples were imaged while in sterile culture in the bioreactor, which simulates in vivo conditions by clamping the tissue radially. For comparison, unclamped sponges were placed on a coverslip. Images were taken of each sample at $1000 \times 1000$ microns and 1000 microns in depth.

\section{Histology}

Standard histology was also performed on tissue samples cultured over the time period they were imaged with the OCM to determine cell distribution, morphology and phenotype. Tissue samples were fixed in a glutaraldehyde-formalin fixative recommended for ophthalmic tissue processing [13] prior to being dehydrated in a series of ethanol solutions. Samples were then soaked in 3:1, 1:1, and 1:3 ethanol:xylene mixtures, respectively. Next, paraffin was introduced and the tissue samples were blocked and sectioned at $5 \mu \mathrm{m}$ on an HM 355E Microm microtome. Sections were then stained with hematoxylin and eosin $(\mathrm{H} \& \mathrm{E})$ to differentiate cell nuclei from collagen matrix.

\section{Scanning Electron Microscopy}

Scanning electron microscopy (SEM) images were taken of sponge samples to assess the microstructure. Samples were coated with gold, mounted on a holder and placed in the SEM chamber. The SEM creates an image of the surface structure of a sample by focusing a beam of electrons onto the sample and collecting those that are backscattered. Images were taken on an ISI Topcon 32B SEM at $15 \mathrm{kV}$.

\section{Sponge Structure}

\section{RESUltS}

We can distinguish differences in microstructure of the collagen sponges with the optical coherence microscope, which can be confirmed with scanning electron microscopy and histology of the same samples. OCM images of less dense sponges with a dense collagen "crust" layer (Fig. 6B) can be compared to those of denser, more homogeneous sponges (Fig. 6C \&D). The "crust" comes from the freeze drying manufacturing process and can be eliminated by modification of the procedure. SEM images confirm the differences in microstructure observed in the OCM. Less dense structure and presence of "crust" can be easily visualized (Fig. 7). Figure 7A shows the sponge structure corresponding to Fig. 6B, and Fig. 7B shows that of Fig. 6C and $\mathrm{D}$. We have also observed that a sponge with cells 
(confirmed with histology) scatters more (has a brighter OCM image) than a sponge without cells (Fig. 8). We hypothesize from the histology results that living cells in the tissue cause more light scattering. We are in the process of doing another study aimed at correlating OCM and histology images directly to test this hypothesis.
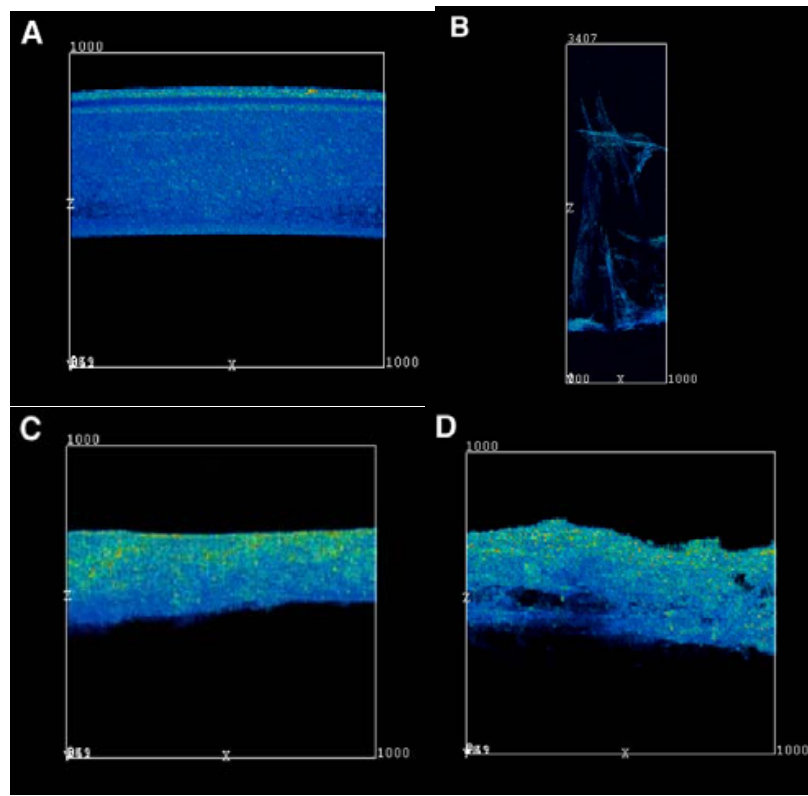

Figure 6. OCM Images of Tissues. Blue indicates relatively low backscatter while red indicates relatively high backscatter. A. Freshly excised rabbit cornea. Image is $1000 \mu \mathrm{m} \times 1000 \mu \mathrm{m}$. B. Unclamped control sponge "crust" (bottom of image) seeded with corneal keratocytes at day 11 in culture. Image is $3407 \mu \mathrm{m} \times 1000 \mu \mathrm{m}$. C. Clamped collagen sponge seeded with cells cultured with ascorbic acid (day 11). Image is $1000 \mu \mathrm{m} x$ $1000 \mu \mathrm{m}$. D. Clamped collagen sponge seeded with cells (day10).Image is $1000 \mu \mathrm{m} \times 1000 \mu \mathrm{m}$. Note the more homogeneous structure of the rabbit cornea and the sponges in $\mathrm{C}$ and $\mathrm{D}$.
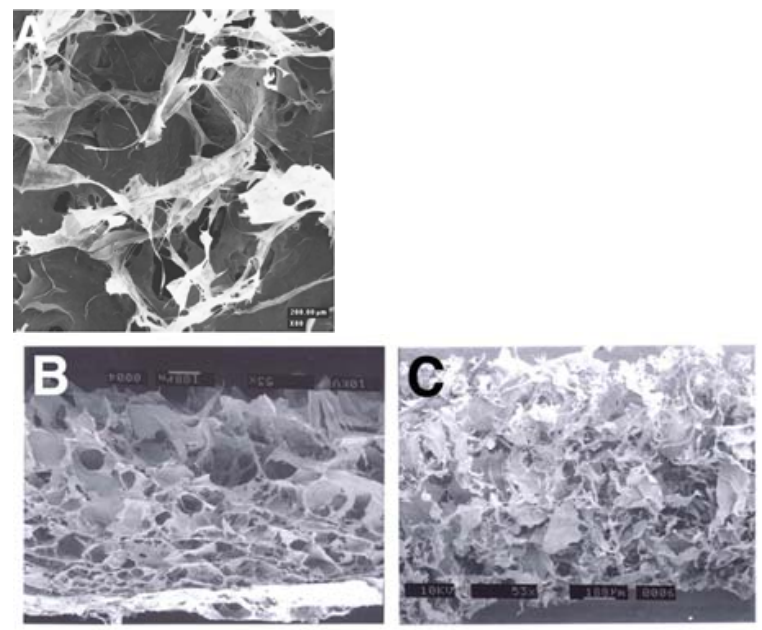

Figure 7: Scanning Electron Microscopy Images. A. Image of collagen sponge with chondroitin sulfate, top view. Structure is less dense and "crust" can be observed at the back of the image. B. Collagen sponge cross-section, illustrating porous structure and "crust" at bottom of image. C. Collagen Sponge with chondroitin sulfate cross-section. Note the lack of "crust" in this sample due to different manufacturing techniques.

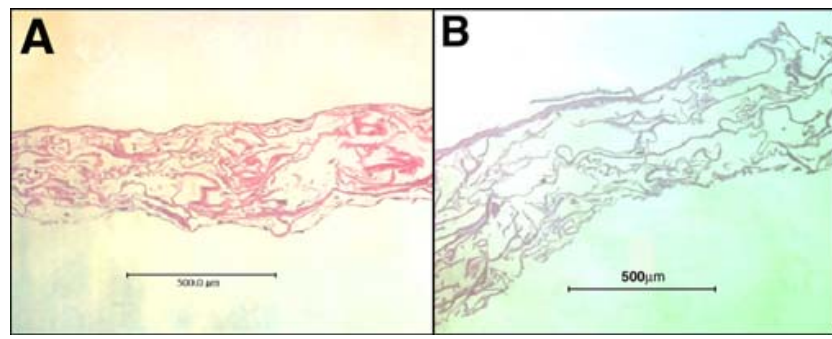

Figure 8: Histology Images of Collagen Sponges where collagen is stained pink with hematoxylin and cell nuclei purple with eosin. A. Image corresponds to Figure 6C. Cells can be observed throughout the sponge. B. Image corresponds to Figure 6D. Note the lack of cells in this sample.

\section{Optical Properties}

In our statistical analysis, we determined the total number of voxels above a certain background voltage, and the summed squared values of these voxels, which is proportional to the intensity of backscattered light from the tissue sample. The amount of backscattered light was quantified in this manner over the xy-plane area of $500 \mu \mathrm{m} \mathrm{x}$ $500 \mu \mathrm{m}$ for the samples tested. Results are shown in Figure 9. The intensities of backscattered light from summer 2002 sponges (Fig 9A) are about five times weaker than that of summer 2003 sponges (Fig. 9B), indicating the greater density of the 2003 samples.

Although both seeded and unseeded sponges are fairly heterogeneous structures, their average optical attenuation coefficient can be determined by calculating the average voxel contents for each $x-y$ plane in the 3D image and plotting them as a function of depth. Fitting this plot to an exponential function yields a rough attenuation coefficient. From the graphs in Figure 10 of average voxel contents versus depth, we can observe light scattering from the epithelial and endothelial layers of the rabbit cornea, and very little scatter in between. In our denser sponge samples (Fig. 6C\&D), there is significant attenuation of the light through the sample, indicating that more light is being scattered. Calculated attenuation coefficients were $7.6 / \mathrm{mm}$ and $12.5 / \mathrm{mm}$ for the two dense sponge structures in different media versus $1.2 / \mathrm{mm}$ in the rabbit cornea.

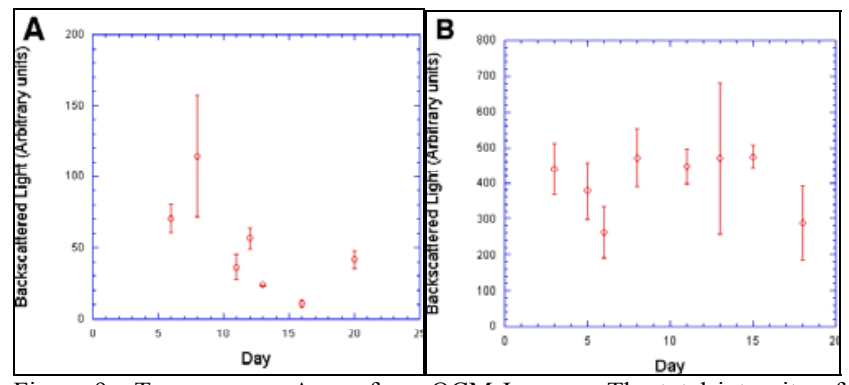

Figure 9: Transparency Assay from OCM Images. The total intensity of backscattered light from the clamped sponges was quantified over the xyplane area of $500 \mu \mathrm{m} \times 500 \mu \mathrm{m}$. Light scattering reflects opacity as well as density of the imaged sample. The intensities of backscattered light from summer 2002 sponges (A) are about five times weaker than that of summer 2003 sponges (B). Samples in A and B correspond to sponge structure in Fig. $6 \mathrm{~B}$ and Fig. $6 \mathrm{C}$ and $\mathrm{D}$, respectively. 


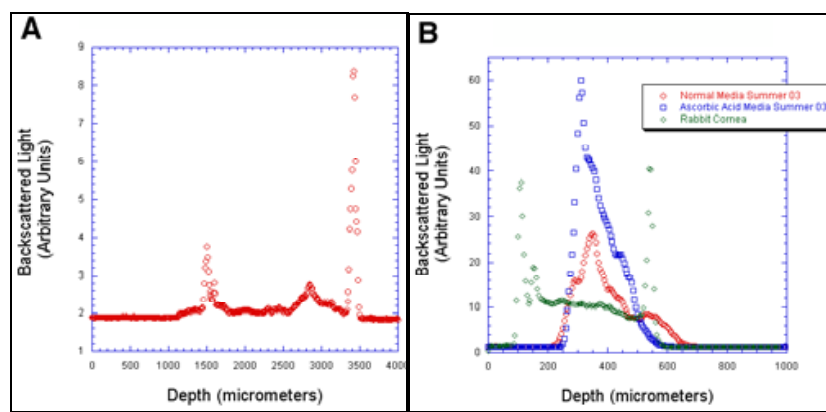

Figure 10: Attenuation Coefficients. The two graphs above compare the amount of backscattered light averaged over a cross-section for a given depth into a tissue sample from the OCM images shown in figure 6. A. The graph shows that the sponge with "crust" (fig. 6B) scatters at the media/sponge interface and at the bottom crust. B. The graph shows a comparison between two sponges with denser structure (fig. 6C\&D) shown in red and blue and a rabbit cornea (shown in green), which attenuate differently.

\section{DISCUSSION}

We have learned a great deal from the analysis of our tissue-engineered corneal constructs with the OCM. The more homogenous, denser structure of sponges is more like that of the rabbit cornea than the less dense sponges with crust. Also, these less dense sponges scatter less than the more homogeneous sponges. The rabbit cornea displays the least backscattering. Cell-seeded collagen sponges attenuate the OCM signal more than the rabbit cornea. OCM data on sponge and cornea microstructure, signal attenuation, and backscatter are internally consistent and corroborate each other. Histology on sponges corroborates OCM image data and links tissue properties to cellular infiltration.

We have learned that we can modulate the optical properties with density of the collagen sponge and can use this information to design an optimal sponge. We need a sponge that is strong enough to withstand tissue culture in tension but not so dense as to increase the scattering properties. We believe that the cells are contributing to higher light scatter in some of the samples, which is consistent with what is known about cell phenotype. Previous studies have shown that cells grown in a collagen sponge express the myofibroblast phenotype in high numbers [9] and this myofibroblast phenotype expressed during corneal wound healing scatters light while the normal unstimulated keratocyte in the transparent cornea does not [10]. These observations have led us to the cell phenotype assessment and modulation studies that are currently underway.

\section{CONCLUSION}

Optical coherence microscopy is a promising method for the evaluation of developing tissue-engineered corneal constructs. It is a non-invasive technique that allows for imaging of the tissue in a controlled and sterile environment. This project significantly enhances the field of corneal tissue engineering in that, if successful, it will provide useful insight into the mechanisms of corneal wound healing. We will be able to determine which factors enhance or detract from the optical properties of our tissue. Our bioreactor/OCM imaging system will allow us to study the effect on transparency of soluble factors, the presence of endothelial cells, the addition of other proteins to the matrix, as well as the application of mechanical forces. The OCM imaging of our tissue constructs may also be helpful in the evaluation of wound healing in the cornea by enabling us to study the structural changes in the tissue constructs over time. The results of this project would prove to be extremely useful in predicting the outcomes of corneal surgeries and vision-enhancing procedures such as LASIK.

\section{ACKNOWLEDGMENT}

We would like to thank the Hubel Lab at the University of Minnesota for providing collagen sponges.

\section{REFERENCES}

[1] Taylor DM, Pamel, GJ. Keratoprosthesis: Possible Utilization in Developing Countries. Refractive and Corneal Surgery. 1991; 7(6): 472-473.

[2] Coster DJ, Williams KA. Transplantation of the Cornea. The Medical Journey of Australia. 1992; 157: 405-408.

[3] Smith GHT, Taylor HR. Epidemiology of Corneal Blindness in Developing Countries. Refractive and Corneal Surgery. 1991; 7(6): 436-439.

[4] Moller-Pedersen T, Cavanaugh HD, Petroll WM, Jester JV. Stromal Wound Healing Explains Refractive Instability and haze Development after Photorefractive Keratectomy: a 1-year Confocal Microscopic Study. Ophthalmology. 2000; 107: 1235-45.

[5] Fini ME. Keratocyte and Fibroblast Phenotypes in the Repairing Cornea. Progress in Retinal and Eye Research. 1999; 18: 529-551.

[6] Doillon CJ, Whyne CF, Brandwein S, Silver FH. Collagen-based Wound Dressings: Control of the Pore Structure and Morphology. Journal of Biomedical Materials Research. 1986; 20: 1219-1228.

[7] Griffith M, Osbourne R, Munger R, et al. Functional Human Corneal Equivalents Constructed from Cell Lines. Science. 1999; 286: 21692172.

[8] Farrell RA, McCalley RL, Tatham PER. Wavelength Dependencies of Light Scattering in Normal and Cold Swollen Rabbit Corneas ands their Structural Implications. Journal of Physiology. 1973; 233: 589612.

[9] Orwin, EJ, Borene, ML and Hubel A. "Biomechanical and Optical Characteristics of a Corneal Stromal Equivalent." Journal of Biomechanical Engineering, 125: 1-6, 2003.

[10] Jester, JV, Moller-Pedersen, T, Huang, J, Sax, CM, Kays, WT, Cavanaugh, HD, Petroll, WM, Piatigorsky, J. (1999) The Cellular Basis for Corneal Transparency: Evidence for "Corneal Crystallins." Journal of Cell Science. 112, 613-622

[11] Zieske JD, Mason VS, Wasson ME, et al. Basement Membrane Assembly and Differentiation of Cultured Corneal Cells: Importance of Culture Environment and Endothelial Cell Interaction. Experimental Cell Research. 1994; 214: 621-633.

[12]Hoeling, BM, Fernandez, AD, Haskell, RC, Huang, E, Meyers, WR, Petersen, DC, Ungersma, SE, Wang, R and Williams, ME. "An optical coherence microscope for 3-dimensional imaging in developmental biology," Optics Express 6:136-146 (March27,2000), (http://www.opticsexpress.org/oearchive/source/19250.htm).

[13] Yanoff M. American Journal of Ophthalmology. 1973; 76:303-304. 